\title{
On Transport of Helium to Grain Boundaries during Irradiation
}

Singh, Bachu Narain; Foreman, A. J. E.

Publication date:

1986

Document Version

Publisher's PDF, also known as Version of record

Link back to DTU Orbit

Citation (APA):

Singh, B. N., \& Foreman, A. J. E. (1986). On Transport of Helium to Grain Boundaries during Irradiation. Risø National Laboratory. Risø-M No. 2612

\section{General rights}

Copyright and moral rights for the publications made accessible in the public portal are retained by the authors and/or other copyright owners and it is a condition of accessing publications that users recognise and abide by the legal requirements associated with these rights.

- Users may download and print one copy of any publication from the public portal for the purpose of private study or research.

- You may not further distribute the material or use it for any profit-making activity or commercial gain

- You may freely distribute the URL identifying the publication in the public portal

If you believe that this document breaches copyright please contact us providing details, and we will remove access to the work immediately and investigate your claim 
RISø-M-2612

ON TRANSPORT OF HELIUM TO GRAIN BOUNDARIES DURING IRRADIATION

Bachu N. Singh and Alan J.E. Foreman *

Abstract. The rate of accumulation of helium at grain boundaries is one of the important parameters determining the integrity and lifetime of the structural components of a fusion reactor. A diffusion calculation is made of the flux of helium to a grain boundary. The $f l u x$ is found to depend on the gas production rate, the width of the cavity denuded zone and the cavity sink strength in the grain interior. The calculated accumulation of helium is in good agreement with the measured gas content of grain boundaries in Al. PEl6 and 316 stainless steel. The $f l u x$ of helium to grain boundaries increases with helium generation rate but the increase is less than proportional to the generation rate. The loss of helium to grain boundaries during the nucleation of the bubbles within the grains has been estimated; no great loss is expected to occur. However,. the loss would be considerably enhanced if any delay in bubble nucleation were to occur due to incubation effects. The role of material variables is found to be difficult to predict at present.

* Materials Development Division

A. E. R. E. Harwel 1

Oxfordshire OXI1 ORA

Engl and

June, 1986

Risø National Laboratory,. DK-4000 Roskilde, Denmark 
This work was presented at the 13th International Symposium on Effects of Radiation on Materials held at seattle. U.S.A, during June 23-25, 1986 .

ISBN 87-550-1275-2

ISSN 0418-6435

Grafisk Service 1986, Risø 
CONTENTS

Page

1. INTRODUCTION $\ldots \ldots \ldots \ldots \ldots \ldots \ldots \ldots \ldots \ldots \ldots \ldots \ldots \ldots \ldots \ldots \ldots \ldots$

2. FLUX OF HEL IUM TO GRAIN BOUNDARIES ............. 6

3. COMPARISON WITH EXPERIMENTS $\ldots \ldots \ldots \ldots \ldots \ldots \ldots \ldots \ldots \ldots$. 8

4. Cavity NUCl Eation MEchanism and hel iUm fluX $\ldots \ldots \ldots \ldots \ldots$

5. ROLE OF IRRADIATION AND MATERIAL VARIABLES ......... 17

6. SUMMARY $\ldots \ldots \ldots \ldots \ldots \ldots \ldots \ldots \ldots \ldots \ldots \ldots \ldots \ldots \ldots \ldots \ldots \ldots \ldots . \ldots . \ldots$

7. REFERENCES ............................. 21

FIGURES $\ldots \ldots \ldots \ldots \ldots \ldots \ldots \ldots \ldots \ldots \ldots \ldots \ldots \ldots \ldots \ldots \ldots \ldots \ldots \ldots \ldots \ldots$ 
1. INTRODUCTION

It is well known that irradiation with $14 \mathrm{MeV}$ neutrons will generate helium at a high rate in the structural materials to be used in fusion devices since helium is practically insoluble in metals and alloys and since the irradiation will take place at relatively high temperatures,. It is inevitable that helium atoms thus generated will diffuse and precipitate as bubbles both in tree grain interior and at the grain boundaries. The continuous accumulation of helium (in the form of bubbles) at grain boundaries will cause grain boundary weakening and embrittlement. Thus, the rate of accumulation of helium at grain boundaries is one of the important parameters determining the integrity and lifetime of the structural components of a fusion reactor. It is, therefore. of interest to understand the processes and parameters controlling the magnitude of helium flux to the boundaries.

In the present paper we first calculate the flux of helium atoms to grain boundaries from the grain interior as well as from the cavity denuded $z$ one along the boundaries; a brief outline of the calculation is given in ref.[1]. This is followed by the consideration of the effect(s) of the major irradiation as well as materials variables on the magnitude of the flux. An evaluation of the role of these variables is,. in our view, essential since the flux is expected to be strongly influenced by the density of sinks at which helium atoms can accumulate (e.g. cavities) and the width of the zone along the boundaries denuded of sinks for helium atoms since all these variables are likely to affect the time/dose dependent evolution of the microstructure in the grain interior and hence the flux of hel ium to grain boundaries, the problem of dose dependence of the flux will be also considered Finallyo. it is pointed out that the knowledge of the helium diffusion behaviour during irradiation is a prerequisite to an understanding of the cavity nucleation mechanism and the evoluation of cavity sink density in the grain interfor which determines the flux of helium atoms to the grain boundaries 


\section{FLUX OF HELIUM TO GRAIN BOUNDARIES}

During irradiation at elevated temperatures where displacement damage and hel ium atoms are generated concurrently, helium atoms would coalesce to form cavities (bubbles/voids). These cavities would then act as sinks for helium atoms produced subsequently. The presence of heterogeneities in the form of clusters of impurity atoms, . precipitates and particles may provide additional sinks for helium atoms. The flux of helium atoms to grain boundaries will be determined by the density and size of sinks for helium in the grain interior and the width of the sink denuded zone along the boundaries

For the sake of simplicity we shall,.in the present treatment, consider cavities to be the only sinks in the grains. Fig. $1(a)$ shows a schematic of cavity distribution. cavity denuded $z$ one and the cavities at the grain boundary. An example of helium accumulation at a grain boundary observed in aluminium irradiated with $600 \mathrm{MeV}$ protons is shown in Fig. 1(b). The micrograph clearly illustrates the presence of well defined denuded zones and a uniform distribution of cavities in the grains

Let us assume that each grain contains a uniform density $c_{c}$ of cavities with mean radius $r^{\prime}$ and a cavity denuded zone of width $d_{z}$ on each side of the boundary. We also assume that the generation of helium is continuous and uniform throughout the whole grain In order to determine the flux of helium to a planar grain boundary we then need to solve the following one-dimensional diffusion equation in the two regions (denuded zone and the grain interior containing cavities):

$$
K_{g}+D \frac{d^{2} c}{d x^{2}}=0 \quad\left(x<d_{z}\right)
$$


$K_{g}+D \frac{d^{2} C}{d x^{2}}-a_{c} D C=0 \quad\left(x>d_{z}\right)$

Where

$$
\begin{aligned}
& x \text { = distance } f \text { rom the grain boundary } \\
& C(x)=\text { helium concentration } \\
& \text { D = helium diffusion coef } f \text { icient } \\
& \mathbf{k}_{\mathbf{g}} \quad=\text { helium generation rate } \\
& d_{z} \quad=\text { denuded } z \text { one width } \\
& \alpha_{c} \quad=\text { cavity sink strength in the grain interior } \\
& =4 \pi r_{c} C_{c}
\end{aligned}
$$

The solution of equations (la) and (lb) is

$$
\begin{array}{ll}
D C=-1 / 2 k_{g} x^{2}+A x & \left(x<d_{z}\right) \\
D C=B e^{-\sqrt{\alpha}} c x+k_{g} / \alpha_{c} & \left(x>d_{z}\right)
\end{array}
$$

Where $A$ and $B$ are constants and are determined from the condition for the continuity of $c$ and $d c / d x$ at $x=d_{z}$.

Thus

$$
\begin{aligned}
& -1 / 2 K_{g} d_{z}^{2}+A d_{z}=B e^{-\sqrt{\alpha_{c}} \dot{d}_{z}}+K_{g} / \alpha_{c} \\
& -K_{g} d_{z}+A=-B \sqrt{\alpha_{c}} e^{-\sqrt{\alpha_{c}} d_{z}}
\end{aligned}
$$

which may be solved for $A$ and $B$. The flux of helium entering the grain boundary is

$$
D\left(\frac{d c}{d x}\right)_{x}=0 \quad=A
$$

so that only A is required Eliminating B from the above equations gives 


$$
A=K_{g} d_{z} \frac{\left(\beta^{1 / 2}+\beta / 2+1\right)}{\beta^{1 / 2(\beta 1 / 2+1)}}
$$

where $\beta=\alpha_{c} d_{z}^{2}$. Thus the total flux $F_{\text {He }}$ from both sides of the grain boundary is

$$
F_{\text {He }}=2 k_{g} d_{z} \frac{\left(\beta^{1 / 2}+\beta / 2+1\right)}{\beta^{l / 2}\left(\beta^{l / 2}+1\right)}
$$

where $\beta=4 \pi r_{c} c_{c} d_{z}^{2}$. The $f l u x F_{H e}$ is independent of the value of the heliun diffusion coefficient, D. The flux of helium calculated from Eg. (6) for $d_{z}=0$ (i.e. no cavity denuded zones) agrees with the results derived previously by Trinkaus [2]. The etfect of denuded zones should not be neglected since their presence causes a noticeable enhancement in the flux (see fig. 2. Ret. (1]).

In order to evaluate the sensitivity of the tlux to the microstructural evolution auring irradiation, the variation of the tlux with cavity density and swelling has been calculated; the results are quoted in Fig. 2. The results show that a significant reduction in the accumulation of helium at the grain boundaries - with a possible reduction in the embrittlement - could be achieved by increasing the cavity density substantially. On the other hand, if the cavity nucleation was to be delayed or suppressed, the flux would be considerably enhanced

\section{COMPARISON WITH EXPERIMENTS}

The validity of the flux calculation can be tested by comparison of the calculated amount of helium accumulated at grain boundaries with the measured amount contained in the grain boundary 
bubbles The amount of helium is determined from the measured size and density of bubbles at grain boundaries. A numerical time integration has been made of eq. (6) for the gas tilux to a boundary in $600 \mathrm{MeV}$ proton irradiated aluminium. The helium bubbles within the grain are assumed to be in equilibrium ( $p=$ $2 Y / r)$ throughout the irradiation and the helium equation of state due to Mills, iriebenberg and Bronson [3] is used The result is found to be almost indentical with that obtained in our previous calculations [1], taking the time average of the bubble size to be 758 of the final size.

The time average of the bubble size can readily be estimated For small highly pressured bubbles it is a reasonable approximation to assume the density of gas atoms in a bubble to be constant during growth so that $r_{c}{ }^{\alpha} t^{1 / 3}$ for a constant bubble density and continuous gas generation Thus, the average size is

$$
\bar{r}_{c}=\int_{0}^{t} r_{c} d t / \int_{0}^{t} d t=3 / 4 r_{c}^{t}
$$

where $r_{c}^{f}$ is the $f$ inal bubble size. A similar result (i.e. $\bar{r}_{c}=$ $2 / 3 \times \frac{t}{c}$ ) can be obtained with the assumption that the gas atoms in the cavities obey the perfect gas 1 aw ( $p v=n k T)$. However, in view of the fact that significant deviations from perfect gas law occur when the gas density in the cavities approaches one vacancy per gas atom, we prefer to use the result of eq. (7). The helium flux to the boundary (given by eg. (6)) is calculated from the measuredvalues of cavity size and density in the grain interior, taking $d_{2}$ to be $1.5 \lambda_{c}$ where $\lambda_{c}$ (cavity spacing) = $\left(c_{c}\right)^{-1 / 3}$. The assumption that $d_{z}=1.5 \lambda_{c}$ is in a reasonable agreement with the measured values of $d_{z}$ in aluminium irradiatea with $600 \mathrm{MeV}$ protons [4].

The experimentally measured values of cavity parameters for high-purity aluminium irradiated with $600 \mathrm{MeV}$ protons at temperatures in the range 120 to $430^{\circ} \mathrm{C}$ are quoted in table $1[5,6]$. From these data, the flux of heli um to grain boundaries has been calculeted and its variation with irradiation temperature is 
silown in Fig 3. Note that Fig 3 does not represent a true temperature dependence of the flux since the irradiation dose (see table 1) is not the same for all the experiments It should be also pointed out that the voids are formed only in a band (of the order of $5 \mathrm{\mu m}$ wide) near the grain boundary; the grain interior must contain a high density of very small (invisible) hel ium bubbles [7]. In the flux calculation for the void case, however, we have assumed that these voids extend into the grain interior and that there is no other sinks for helium in the grain interior. This simulates the situation observed in aluminium irradiated with fast neutrons with a rather low helium generation rate $[8]$.

For comparison, the experimentaliy measured tiluxes of hel ium to grain boundaries in high-purity al uminium irradiated with 600 $\mathrm{MeV}$ protons at temperatures in the range 120 to $220^{\circ} \mathrm{C}$ are also quoted in Fig. 3. It can be seen that the calculated fluxes agree quite well with the measured values The results quoted in Fig. 3 clearly demonstrate that the rate of helium accumulation at grain boundaries during $600 \mathrm{MeV}$ proton irradiation of aluminium would have been an order of magnitude greater if only a normal density of voids had been nucleated throughout the whole grain It should be noted that the increase in the flux with increasing temperature is entirely due to changes in $\beta(=$ $4 \pi r_{c} c_{c} d_{z}^{2}$ ). Thus, these results clearly demonstrate that even for c. given helium generation rate and helium diffusion mechanism. the rate of helium tlow to a boundary will be seriously affected by the maynitude of $\beta$ generated during irradiation (see later for further discussion). 
Table 1. Cavity size, density and swelling in the grains of aluminium irradiated at different temperatures with $600 \mathrm{M} \in \mathrm{V}$ proton.

a) Voids [5].

\begin{tabular}{lcccc}
\hline $\begin{array}{l}\text { Irradiation } \\
\text { Temp. } \\
\left({ }^{\circ} \mathrm{C}\right)\end{array}$ & Dose & Mean Diam & Density & Swelling \\
\hline & $(\mathrm{dpa})$ & $(\mathrm{nm})$ & $\left(\mathrm{m}^{-3}\right)$ & $(8)$ \\
120 & 2.5 & 54 & $1.7 \times 10^{19}$ & $\left.0.140^{\star}\right)$ \\
130 & 5 & 67 & $1.1 \times 10^{19}$ & $\left.0.173^{\star}\right)$ \\
140 & 5 & 83 & $3.5 \times 10^{18}$ & $\left.0.104^{\star}\right)$
\end{tabular}

b) Bubbles [6].

\begin{tabular}{lcccc}
130 & 5 & 3 & $7.5 \times 10^{22}$ & 0.105 \\
140 & 5 & 3 & $6.0 \times 10^{22}$ & 0.085 \\
170 & 3 & 4.1 & $3.1 \times 10^{22}$ & 0.111 \\
220 & 2.5 & 6.9 & $4.5 \times 10^{21}$ & 0.077 \\
318 & 1.4 & 16.8 & $6.1 \times 10^{20}$ & 0.154 \\
430 & 5 & 85.1 & $7.0 \times 10^{19}$ & 2.250 \\
\hline
\end{tabular}

* assuming the presence of voids in the whole grain.

Cavity parameters for grain interiors as well as grain boundaries have been reported for PEI6 (9) and AISI 316 stainless steel [10]; the relevant data are quoted in Table 2. Table 3 gives the comparison between the measured and the calculated helium contents of the grain boundaries in Al (99.99998), PE 16 and AISI 316 stainless steel. The agreement for Al and 316 
Table 2. Densities and mean diameters of cavities in grains and grain boundaries

\begin{tabular}{cccccc}
\hline & $\begin{array}{c}\text { Irradiation } \\
\text { Material }\end{array}$ & $\mathrm{T}^{\mathrm{T}} \mathrm{T}_{\mathbf{M}}$ & \multicolumn{2}{c}{ Grain interior } & \multicolumn{2}{c}{ Grain Boundary } \\
& $\left({ }^{\circ} \mathrm{C}\right)$ & Density & Diam. & Density & Diam. \\
& & $\left(\mathrm{m}^{-3}\right)$ & $(\mathrm{nm})$ & $\left(\mathrm{m}^{-2}\right)$ & $(\mathrm{nm})$ \\
\hline
\end{tabular}

$\begin{array}{lllllll}\text { aAla } & 220 & 0.53 & 4.5 \times 10^{21} & 6.9 & 2.7 \times 10^{14} & 11.0\end{array}$

$\begin{array}{llllll}625 & 0.52 & 3.3 \times 10^{22} & 2.8 & 2.0 \times 10^{15} & 3.5\end{array}$

PE $16^{\text {b) }}$

625

$0.526 .6 \times 10^{22}$

$3.21 .2 \times 10^{15}$

6.0

625

$0.52 \quad 2.7 \times 10^{22}$

$6.32 .0 \times 10^{15}$

5.5

$\begin{array}{lllllll}\text { AISI } 316^{c} \text { ) } & 700 & 0.56 & 2.0 \times 10^{20} & 14.0 & 1.2 \times 10^{15} & 8.4\end{array}$

a) irradiated with $600 \mathrm{MeV}$ protons, Ret. [6].

b) irradiated simultaneously with nickel ions, $\alpha$-particles and deutrons, Ret. [9]. Note that the larger cavities have been excluded since their effect on the flux would be insignificant.

c) helium implanted homogeneously, Ref. [10].

stainless steel is very good In the case of PE 16, the calculation overestimates the amount of helium reaching the boundaries by a factor of 2-3. It should be noted, however, that PEl 6 contains a dense $\sim 10^{22} \mathrm{~m}^{-3}$, population of small $\sim 15 \mathrm{~nm}$ precipitates of $\gamma^{\prime}$ phase. In addition, these specimens contained dislocations in the form of a tight network [9]. Both these factors would be expected to enhance the trapping and retention of belium within the grains, sausing a reduction in the flux of 
helium to grain boundaries For instance, the presence of coldwork microstructure in Al-Mg alloy irradiated with $800 \mathrm{MeV}$ protons has been fousd to reduce the gas flux by a factor of $\sim 2-5$ [11]; both types of specimens were irradiated under identical conditions.

4. CAVITY NUCLEATION MECHANISM AND HELIUM FLUX

It is clear from the previous section that the flux of helium to grain boundaries calculated $f$ rom eq. (6) agrees very well with the experimental results No doubt, eq (6) can be used aiso for predicting the flux to the boundaries but only if the evolution of the cavity (or any other sink) parameters in the given material under the given irradiation conditions could be reliably predicted.

In order to predict the magnitude of the cavity parameters, it is essential, however, that the cavity nucleation mechanism is reasonably well understood since it is the nucleation mechanism that determines the scale of cavity nucleation $\left(c_{c}\right)$ and the width of the cavity denuded zones $\left(d_{z}\right)$. In addition, during early stages of irradiation, the magnitude of the flux will be affected by the rate of cavity nucleation because of continuous changes in both $c_{c}$ and $d_{2}$ until the nucleation comes to saturate Thus, it is clear that the flux of helium calculated from eg. (6) would be an underestimate of the flux reaching the boundary during early stages of irradiation It is possible, however, to evaluate the magnitude of this extra 1088 of gas to the boundaries since the bubbles within the grains are extremely small at the nucleation stage (i.e $\beta$ is small), eg. (6) can be modified to

$$
F_{H e}=2 K_{g} d_{z} / \beta^{l / 2}=2 k_{g} /\left(4 \pi r_{c} c_{c}\right)^{1 / 2}
$$

which is independent of the width of the cavity denuded zone 
Computer calculations of the diatomic nucleation of helium bubbles [12] show that the cavity density $c_{c}$ increases almost linearly with time, until it attains a value within a factir 2 of the saturation density, whilst the average cavity size remains al most unchanged during this period. Thus, the mean flux of helium to a boundary during the nucleation time $\tau$ is

$$
F_{\text {He }}^{\text {mean }}=\frac{1}{\tau} \int_{0}^{\tau} F_{H e} d t=2 F_{\text {He }}^{\tau}
$$

since $F_{\text {He }} \propto t^{-1 / 2}$ (from eq. (8)), where $F_{\text {He is the flux at }}^{\text {T }}$ the ena of the nucleation period $\tau$. The effect of the steadily increasing cavity density is thus to double the flux of helium to the boundaries during this period

Diatomic nucleation calculation for $800 \mathrm{MeV}$ proton irradiated aluminium shows that the linear increase is completed in $<0.01$ dpa at a temperature of $70^{\circ} \mathrm{C}$ Taking $K_{g}$ to be $335 \mathrm{appm} / \mathrm{dpa}$, we estimate that the gas loss to a boundary during this period is enhanced by $<5 \times 10^{16}$ at $\mathrm{m}^{-2}$ due to the 1 inear increase in cavity density within the grains. This represents a relatively small contribution to the gas content of a boundary, which is typically in excess of $10^{18}$ at. $m^{-2}$ (Table 3 ).

The accumulation of gas on a grain boundary can, however, be substantially increased, if there is an incubation period before the nucleation of cavities within the grains is initiated for example, with a grain diameter of $\sim 10 \mu \mathrm{m}$ and no dislocations or other sinks within the grain a helium generation rate of 335 appm/apa could deposit $7 \times 10^{18}$ at. $^{-2}$ of helium on the boundary in only 0.1 dpa. The presence of any internal sinks such as dislocations would, of course, be expected to reduce the effect of the incubation 
Table 3. Measured and calculated helium content of giain boundaries.

\begin{tabular}{|c|c|c|c|c|c|}
\hline \multirow[t]{2}{*}{ Material } & \multirow{2}{*}{$\begin{array}{l}\text { Helium generation } \\
\text { o implantion rate } \\
\left.\text { (at. } / \mathrm{m}^{3} / \mathrm{s}\right)\end{array}$} & \multirow{2}{*}{$\begin{array}{l}\text { Irradiation } \\
\text { Temperature } \\
\text { (ब) }\end{array}$} & \multirow[t]{2}{*}{$T / T_{M}$} & \multicolumn{2}{|c|}{$\begin{array}{r}\text { Grain boundary helium } \\
\text { content }\left(a t / m^{2}\right)\end{array}$} \\
\hline & & & & Measuredb) & Calculated ${ }^{c)}$ \\
\hline$A \perp$ & $4.5 \times 10^{19}$ & 220 & 0.53 & $5.9 \times 10^{18}$ & $6.9 \times 10^{18}$ \\
\hline
\end{tabular}
4. $5 \times 10^{21 a)}$
625
0.52
$2.8 \times 10^{18}$
$9.6 \times 10^{18}$

FE16

$\begin{array}{lllll}3.5 \times 10^{21 a} & 625 & 0.52 & 6.3 \times 10^{18} & 1.2 \times 10^{19}\end{array}$

$$
\text { 4. } 1 \times 10^{21 a)}
$$

625

0.52

$8.5 \times 10^{18}$

$2.9 \times 10^{19}$

$\begin{array}{llllll}\text { AISI } 316 & 2.4 \times 10^{21} & 700 & 0.56 & 1.4 \times 10^{19} & 1.7 \times 10^{19}\end{array}$
a) assuming an average damage rate of $4.5 \times 10^{-3}$ dpa $8^{-1}$.
b) using the helium equation of state from Mills et al [3].
c) using only 758 of the observed cavity size in the grain interior quoted in table 2; see text for explanation.

In any gas-assisted cavity nucleation mechanism the scale of nucleation will be controlled by the rate of gas (i.e helium) diffusion under the given set of irradiation conditions Thus, a knowledge of helium diffusion behaviour during irradiation is a prerequisite to an understanding of the nucleation kinetics and the dose dependence of the flux to grain boundaries.

Recently we have investigated the problems of helium diffusion and cavity nucleation [12]. In this investigation the mechanism for helium diffusion is considered to be the normal mechanism 
for the radiation enhanced diffusion of substitutional atoms, where the addition of a second vacancy enables the trapped nelium atom (in a single vacancy) to make one or more jumps Detailed computer calculations of diatomic nucleation by this diffusion mechanism have been made [12] assuming the helium diffusivity to be proportional to $C_{v} D_{v}$ where $C_{v}$ is the vacancy concentration and $D_{v}$ the vacancy diffusivity.

These calculations show that above $\sim \mathbf{T}_{\mathfrak{m}} / 2$ the bubble density has an activation energy of $E_{s d} / 2$, where $E_{s d}$ is the self-diffusion energy. This is in excellent agreement with the experimental results for $600 \mathrm{MeV}$ proton irradiated aluminium [6]. For temperatures below $\sim \mathrm{T}_{\mathrm{m}} / 2$ the bubble density should have an activation energy of $E_{m}^{V} / 3$, where $E_{m}^{V}$ is the vacancy migration energy. This is also consistent, at least qualitatively, with the experimental results for $600 \mathrm{MeV}$ proton irradiated aluminium [6]. A significant reduction in the activation energy is observed below $\sim T_{m} / 2[6]$, although there is yet insuficient data at lower temperatures to determine it this $\mathrm{falls}$ to $\mathrm{E}_{\mathrm{m}}^{\mathrm{V}} / 3$.

Above $\sim_{m} / 2$, the bubble density is predicted to be proportional to the square root of the helium generation rate (per unit time) and to be independent of damage rate. At temperatures below $\sim \mathbb{T}_{\mathrm{m}} / 2$ the bubble density is predicted to vary also as the square root of the helium generation rate (per unit time) but inversely as the one-quarter power of the damage rate unfortunately, there is not sufficient experimental data for a quantitative verification of the predicted dependencies on the helium generation rate and damage rate in the whole temperature range How ever, as far as the effect of helium generation rate on cavity density is concerned, the limited results available at present $[9,13,14]$ are not inconsistent with the predictions. Thus, it seems possible that using the diatomic nucleation in terms of radiation enhanced diffusion of helium, the flux of helium to grain boundaries can be predicted with the help of eqs. $(6,8,9)$. It should be emphasized, however, that the present diatomic nucleation model does not take into account the effects of material variables. 
5. ROLE OF IRRADIATION AND MATERIAL VARIABLES

The gas flux to grain boundaries and its dose dependence would be affected by material and irradiation variables since they control the rate, magnitude and type of sinks (for helium) evolution during irradiation since the effect of these variables on the nucleation and growth behaviour of cavities (or any other sink) cannot at present be predicted with any accuracy, in the following, we examine the effect of some of these variables on the flux of heliun to grain boundaries determined experimental$1 y$.

Table 4 shows experimental results on the flux of helium to

Table 4. Effect of irradiation and material variables on helium flux to grain boundaries

\begin{tabular}{|c|c|c|c|c|c|c|c|}
\hline Material & $\begin{array}{l}\text { Irr. } \\
\text { Texp } \\
\left({ }^{\circ} \mathrm{C}\right)\end{array}$ & $T / T_{M}$ & $\begin{array}{c}F_{\mathrm{Be}} \\
\left(\mathrm{at} \mathrm{m}^{-2} \mathrm{~s}^{-1}\right)\end{array}$ & $\begin{array}{l}\text { Dose } \\
\text { (dpa) }\end{array}$ & $\begin{array}{c}\mathrm{Kg} \\
\left(\mathrm{at} \mathrm{m}^{-3} \mathrm{~s}^{-1}\right)\end{array}$ & $\begin{array}{l}\mathrm{F}_{\mathrm{He}} / \mathrm{K}_{\mathrm{g}} \\
\left(10^{-8_{m}}\right)\end{array}$ & Ref. \\
\hline Ai & $40-100$ & $0.33-0.40$ & $4.7 \times 10^{11}$ & 0.2 & $1.6 \times 10^{18}$ & 29 & 11 \\
\hline $\mathbf{A}$ & 120 & 0.42 & 4. $1 \times 10^{12}$ & 0.6 & 4. $5 \times 10^{19}$ & 9.1 & 15 \\
\hline $\mathbf{A}$ & 220 & 0.53 & $8.3 \times 10^{12}$ & 2.5 & $4.5 \times 10^{19}$ & 18.4 & 6 \\
\hline PE16 & 625 & 0.52 & $1.6 \times 10^{14}$ & 80 & $4.5 \times 10^{21}$ & 3.6 & 9 \\
\hline $3165 S$ & 700 & 0.56 & $1.3 \times 10^{15}$ & 0.04 & 2. $4 \times 10^{21}$ & 54.2 & 10 \\
\hline
\end{tabular}


grain boundaries, $F_{H e^{\prime}}$ and $F_{H e} / K_{g}$ (where $K_{g}$ is helium generation rate) for different materials obtained under different irradiation conditions $[6,9-11,15]$. As mentioned earlier, the amount of helium transported to grain boindaries is determined from the measured size and density of bubbles at the grain boundaries. since the irradiation dose used in these experiments are known, $F_{\text {He }}$ values are easily calcu. ated.

\section{(a) Effect of Helium Generation Rate}

According to eq. (6), the helium generation rate, $K_{g^{\prime}}$ is expected to affect $F_{H e}$ directly as well as indirectly: the indirect effect coming via changes in $\beta$ and $d_{2}$ since the cavity density is likely to vary with $\mathrm{K}_{\mathrm{g}}[16]$. The ratio $\mathrm{F}_{\mathrm{He}} / \mathrm{K}_{\mathrm{g}}$ represents the magnitude of the indirect effect due to changes in $B$ and $d_{z}$. Assuming that the cavity nucleation-time is short and that it does not change with $\mathbb{R}_{g^{\prime}}$ the ratio $F_{H e} / K_{g}$ would be expected to decrease with increasing $\mathrm{K}_{\mathrm{g}}$

Experimental data quoted in Table 4 do in fact show that $F_{\text {He }}$ increases and $F_{H e} / K_{g}$ decreases with increasing hel ium generation rate In high-purity aluminium irradiated with 600 and $800 \mathrm{MeV}$ protons, an increase in $k_{g}$ by a factor of $\sim 28$ leads to an increase in $F_{\text {He }}$ only by a factor of $\sim 10$. In other words, the microstructure evolved under $800 \mathrm{MeV}$ proton irradiation (low $\mathbf{k}_{\mathbf{g}}$ ) is only about one-third as effective in trapping the helium atoms within the grains as is the case under $600 \mathrm{MeV}$ proton irradiation (high $\mathrm{K}_{\mathrm{g}}$ ). The lower sink strength in the case of $800 \mathrm{MeV}$ proton irradiation could easily arise due to lower $\mathrm{K}_{\mathrm{g}}$ since $C_{c}$ is calculated to be proportional to $\left(K_{g}\right) 1 / 2[16]$. A comparison of the results for aluminium irradiated at $0.53 \mathrm{~T}_{\mathrm{m}}$ with that for PEl6 irradiated at $0.52 \mathrm{~T}_{\mathrm{m}}$ al so show s that $F_{\text {He }}$ increases and $F_{H e^{/ K}}$ decreases with increasing $K_{g}$; as can be seen in Table $2, c_{c}$ (in the grain interior) increases approximately as $\left(k_{g}\right)^{1 / 2}$. 
(b) Alloying Fifects

The effect of alloying on gas accumulation at grain boundaries is expected to be even more complicated than that of helium generation rate. The complication is likely to arise from the fact that alloying additions may modify not only the point defect concentration and sink-strength (cavities and precipitates) in the grain interior but may also affect the diffusion properties of gases Consequently, alloying may enhance [17] or suppress the nucleation of cavities $[18,19]$. This impiies that if an alloying addition was to suppress the nucleation and growth of cavities, the gas flow to grain boundaries in such a material could be vastly enhanced (in the absence of highdensity of precipitates) or could be brought down to zero if the material contained a high density of small precipitates [20].

Two recent investigations on aluminium-alloys $[11,21]$ have demonstrated dramatic effects of alloying on the amount of gas accumulated at grain boundaries in AlMg3 and Al-Mg-Si alloys. For example, during $800 \mathrm{MeV}$ proton irradiation [11], the amount of gas accumulated at the grain boundaries in AlMg3-alloy is about an order of magnitude higher than at the grain boundaries in the high-purity aluminium irradiated under identical conditions. In the Al-Mg-Si alloy, on the other hand, no bubbles could be resolved at the grain boundaries. In both cases, no cavities were found in the grain interior. In the high-purity AI-Mg-Si alloy irradiated with $600 \mathrm{MeV}$ protons, however, a high density of bubbles were observed at the grain boundaries [2l]. Furthermore, the amount of gas accumulated at the boundaries in the high-purity $A \perp-M g-S i$ alloys was considerably greater than that found in high-purity aluminium irradiated with $600 \mathrm{MeV}$ protons. These results clearly illustrate the limitations of eqs. $(6,8,9)$ in predicting the gas flux to grain boundaries in materials in which neither the diffusion of gases nor the nucleation and growth behaviour of cavities and precipitates are well understood

Thus, the experimental observations presented in this section show that the effects of irradiation and material variables on 
microstructural evolution are rather complicated on balances it seems as if it is not terribly difficult to deal with the etfects of helium generation rate (when considered in terms of per unit time and not d(a). However, it appears to be almost impossible at present to predict the effect of material variables and of changes in material variables during irradiation (eg microchemistry, phase stability, etc.) on the rate of helium transport to grain boundaries These results also expose some of the difficulties involved in the selection of strategy for so called "simulation experiments".

\section{SUMMARY}

The accumulation of irradiation produced helium or any other gas atoms in the form of bubbles at grain boundaries can cause weakening and embrittlenent of a material. A calculation has been made of the flux of helium to a grain boundary, taking into account the cavity denuded $z$ one and the cavity sinks for helium within the grains. The flux is found to depend on the gas production rate and the cavity sink strength in the grain interior.

The validity of the calculations is tested by comparison with the helium content measured on grain boundaries in high-purity al umini um, PEl6 and 316 stainless steel irradiated in different tacilities. The overall agreement is shown to be good for these materials The gas flux calculations demonstrate that the accumulation of helium on the boundaries can be reduced by $1-2$ orders of magnitude if a high density of gas bubbles is nucleated within the grains. The flux of helium to grain boundaries increases with increasing helium generation rate but the increase is less than proportional to the generation rate because of the effect of generation rate on cavity sink strength.

The flux of gas to the boundaries will be enhanced during the nucleation of bubbles within the grains An estimate of this 
loss on the basis of diatomic nucleation indicates that because of fast nucleation no great loss of gas should occur during this type of nucleation However, the gas loss to the boundaries could be considerably enhanced if any delay in bubble nucleation were to occur due to incubation effects.

It is argued that any irradiation and/or material variables affecting the sink strength for helium in the grair interior would modify the gas flux to the boundaries. It is pointed out that the tlux of helium to grain boundaries is dependent primarily on the nucleation behaviour of cavities or/and any other sink in the grain interior.

It seems possible that using the diatomic nucleation in terms of radiation enhanced diffusion of helium, the effect of helium generation rate on the flux of helium to grain boundaries could be predicted with the help of eqs. $(6,8,9)$. It should be emphasized, however, that the prediction would be 1 imited only to those cases where the diffusion of helium and the nucleation behaviour of helium bubbles are reasonably well understood.

Experimental results clearly demonstrater on the other hand, that the effect of material variables on the flux of gas atoms to grain boundaries is very complicated. In order to predict the flux, the effect of material variables on gas diffusion and on bubble nucleation and growth will have to be investigated.

\section{REFERENCES}

(1) Foreman, A. J. E. and Singh, B. $M$, Journal of Nuclear Material s, vol. 133 \& 134,1985, p. 451.

[2] Trinkaus, H, Journal of Nuclear Materials, vol, 118, 1983, p 39.

[3] Mills, R. L. , Liebenberg, D. H. and Bronson, J. C., Pbysical Review, vol. B 21, 1980, p 5137. 
[4] Singh, B N, Leffers, T. L. Green, W. V. and Victoria, H. Journal of Nuclear Materials, vol. 122 \& 123 , 1984, a 703.

[5] Victoria, M, Green, W. V., Singh, B $\mathbf{N}$ and Leffers, T., Effects of Radiations on Materials: 13 th Symposium, ASTM special Technical Publication, in press

[6] Victoria, H. Green, W. V., Singh, B. $\mathbf{N}$ and Leffers, T., Journal of Nuclear Materials, vol. $122 \& 123,1984, p$ 737.

17] Singh, B. N. Leffers, T. Green, W. V. Green, S. L. Journal of Nuclear Materials, vol. 105, 1982, p. 1.

[8] Hor sewell, $A$ and Singh, $B N$, Effects of Radiations on Materials: ASTM Special Technical Publication 870 (American Society for Testing and Materials, Philadelphia), 1985, p. 248.

[9] Parrell, $\mathrm{K}$, and Packan, $\mathrm{N} H$, Journal of Nuclear Materials, vol. $103 \& 104,1981$, p. 1059.

[10] Ullmaier, H, Journal of Nuclear Materials, vol. 133 134,1985, a 100.

[11] Singh, B N, Hor sewel,, A, Sommer, W. F. and Lohmann, W. , Second International Conference on Fusion Reactor Materials, Cnicago, April 1986, Journal of Nuclear Materials, in press.

[12] Foreman, A J. E and Singh, $B$, in Ref. 11.

[13] Briminall, J. Lo and simonen, E P., Journal of Nuclear Materials, vol. 68, 1977, p 235.

[14] Gavillet, D, Gotthardt, R, Martin, J.-L, Green $S$ L, Green, W. V. and Victoria, $M$, in Ref. 8, p 394.

[15] Singh, B $\mathbb{N}$, Leffers, T., Green, W. V. and Victoria, $M$, unpubl ished wor $k$.

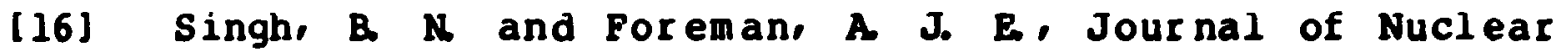
Materials, vol. 103 104, 1981, po 1469.

[17] Singh, B $\mathbb{N}$, Leffers, T. and Barlow, R., Proc of International Conf, "High Voltage Electron Microscopy 1977\%, Japanese Society of Electron Microscopy, Tokyo, 1977, p 581.

[18] Singh, B $M$, Leffer B, T., Makin, M. J., WalterB, G P. and Foreman. A J. E., Journal of Nuclear Materials, vol. $103 \leftarrow 104,1981, p 1041$. 
[19] Farrell, K, Journal of Nuclear Materials, vol. 97, 1981, p. 33.

[20] Kesternich, W. and Rothaut, J., Journal of Nuclear Materials, vol. $103 \& 104,1981$, p 845.

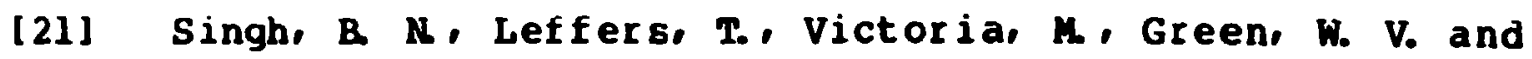
Gavillet, D., in Ret. 11 . 

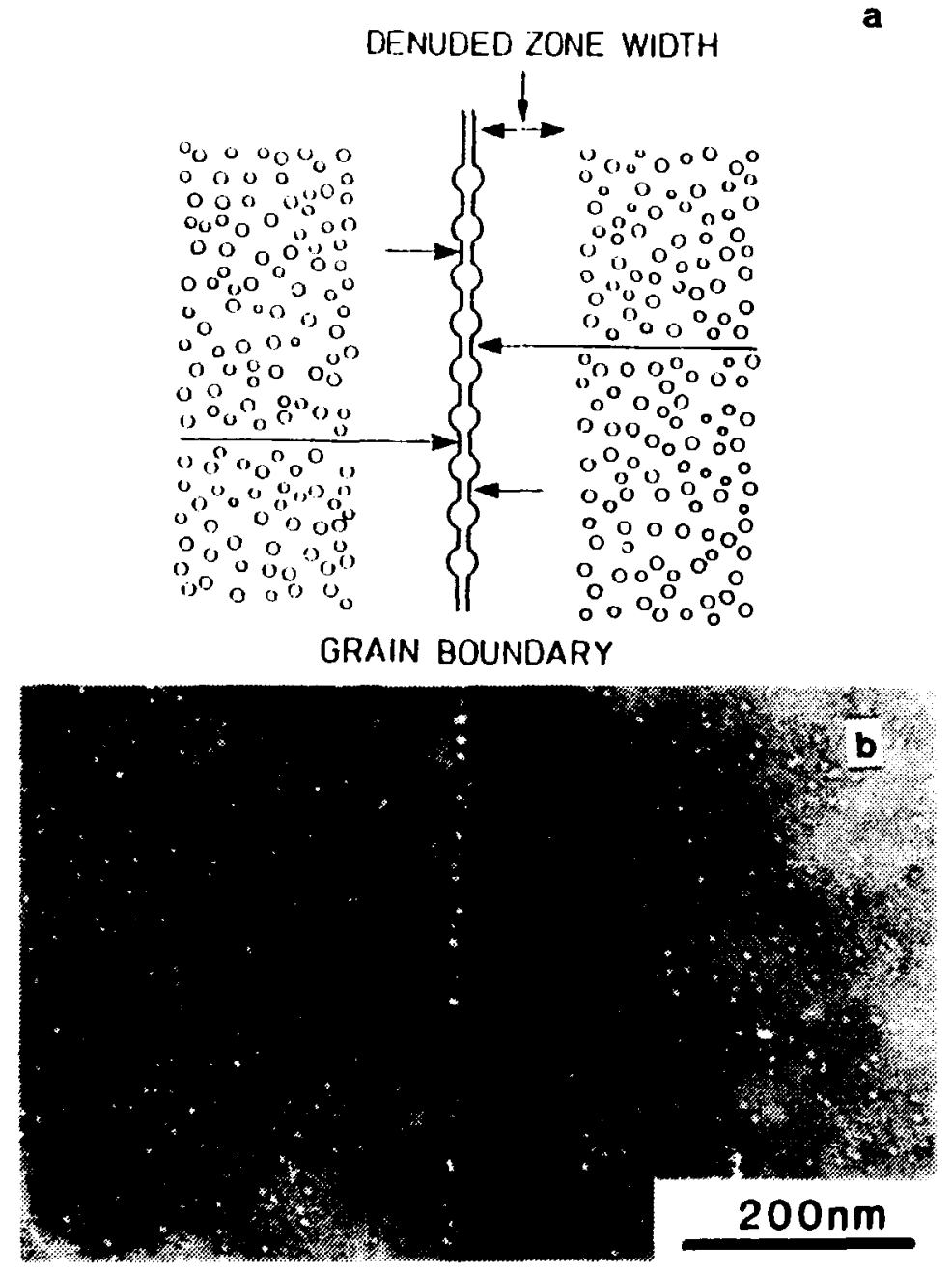

Eige 1. (a) Schematic of helium transport to a grain boundary from the denuded zone and the grain interior. (b) Precipitation of helium in the form of bubbles within grains and at a grain boundary in high-purity aluminium irradiated with $600 \mathrm{MeV}$ protons at $220^{\circ} \mathrm{C}$ to a dose of 2.5 dpa. 


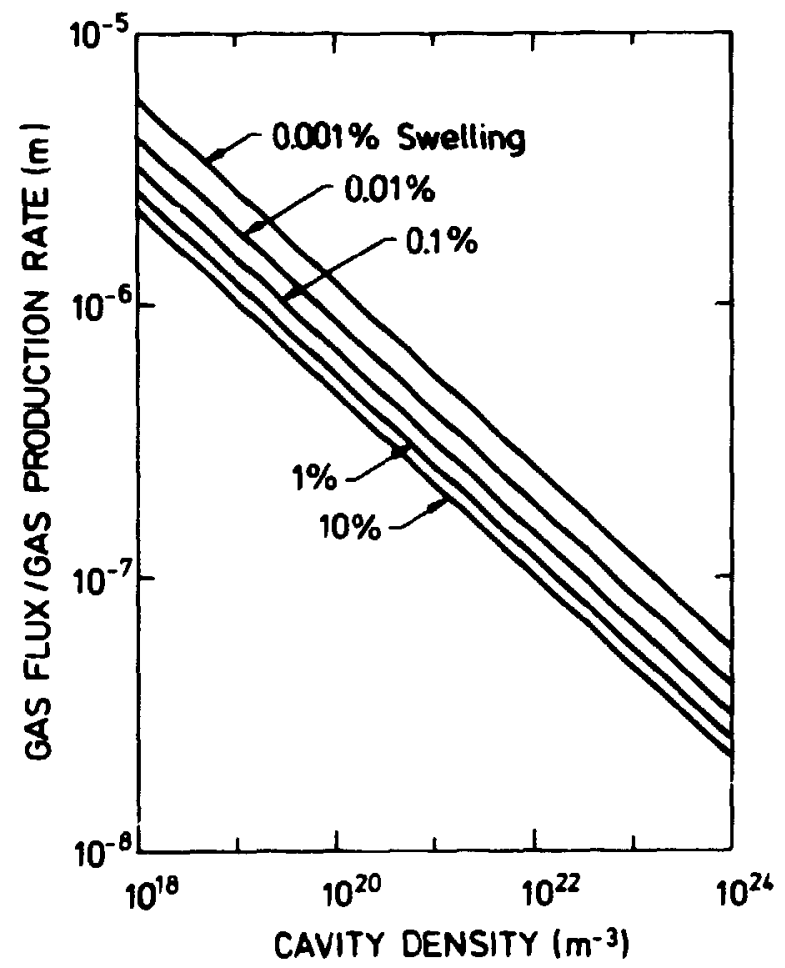

Eig. 2. Calculated helium flux to a grain boundary as a function of density and sink strength of cavities within the grains on both sides of the boundary. The denuded zone width is taken to be $1.5 \lambda_{c}$ where $\lambda_{c}$ is the mean cavity spacing 


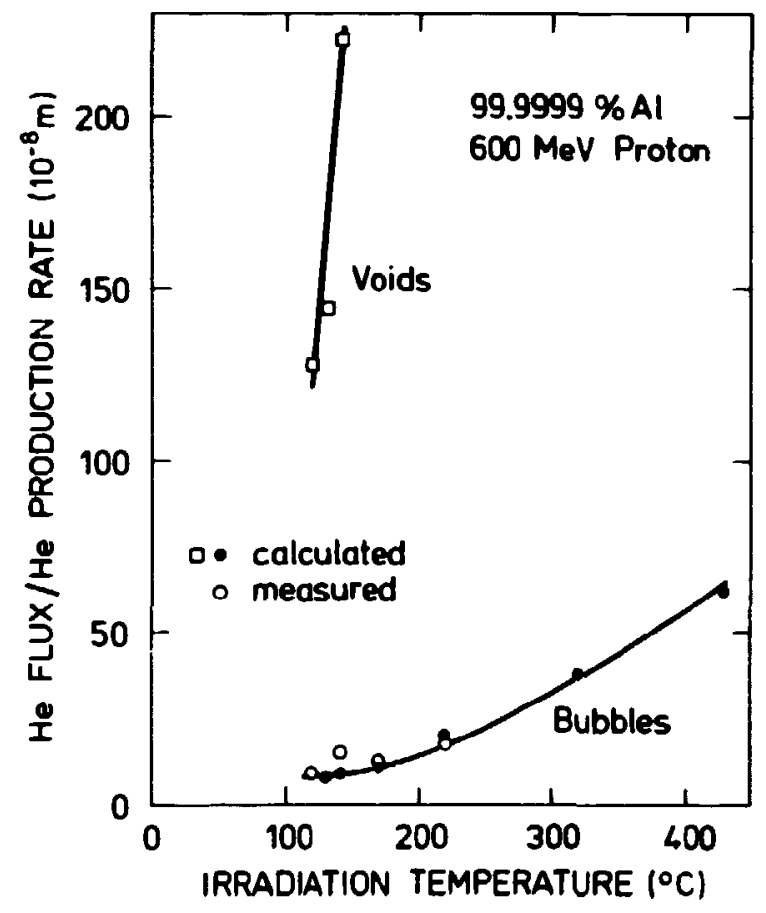

Eig. 3. Calculated and measured helium tlux to a grain boundary in high-purity aluminium irradiatea with $600 \mathrm{MeV}$ protons as a function of irradiation temperature. (See Table 1 for cavity parameters $[5,6])$. 


\begin{tabular}{|c|c|}
\hline \multirow{2}{*}{$\begin{array}{l}\text { Title and author(s) } \\
\text { On transport of helium to grain boundaries } \\
\text { during irradiation }\end{array}$} & Date June 1986 \\
\hline & $\begin{array}{l}\text { Department or group } \\
\text { Metallurgy Department }\end{array}$ \\
\hline & Groups own registration number(s) \\
\hline & Project/contract no. \\
\hline Tables 4 Illustrations 3 Reforences 21 & ISBN $87-550-1275-2$ \\
\hline
\end{tabular}

Abstract (Max. 2000 char.)

The rate of accumulation of helium at grain boundaries is one of the important parameters determining the integrity and lifetime of the structural components of a fusion reactor. A diffusion calculation is made of the flux of helium to a grain boundary. The flux is found to depend on the gas production rate, the width of the cavity denuded zone and the cavity sink strength in the grain interior. The calculated accumulation of helium is in good agreement with the measured gas content of grain boundaries in Al, PE16 and 316 stainless steel. The flux of helium to grain boundaries increases with helium generation rate but the increase is less than proportional to the generation rate. The loss of helium to grain boundaries during the nucleation of the bubbles within the grains has been estimated; no great loss is expected to occur. However, the loss would be considerably enhanced if any delay in bubble nucleation were to occur due to incubation effects. The role of material variables is found to be difficult to predict at present.

Descriptors - INIS

ALUMINIUM; AUSTENITIC STEELS; BUBBLES; BUILDUP; CAVITIES; DIFFUSION; DISLOCATIONS; GRAIN BOUNDARIES; HELIUM GENERATION; IRRADIATION; NUCLEATION; PROTON BEAMS; STEEL-CRI7NI12MO3; THERMONUCLEAR REACTOR MATERIAL; VOIDS 